\title{
HIGH RESOLUTION TECHNIQUE FOR MEASURING CHARGE AND POLARIZATION DISTRIBUTIONS IN DIELECTRICS BY PIEZOELECTRICALLY INDUCED PRESSURE STEP WAVES (PPS)
}

\author{
M. Haardt, W. Eisenmenger \\ Physikalisches Institut der Universität Stuttgart \\ D-7000 Stuttgart 80, Pfaffenwaldring 57, Germany.
}

Experimental technique

In the past, several experimental methods have been developed [1] for the analysis of charge and polarization distributions within dielectrics. The acoustic pressure step method originally proposed by Laurençeau et al. [2] recently could be improved significantly achieving resolutions in the order of a few $\mu \mathrm{m}[3,4]$. Instead of using pressure steps generated by a shock tube [2] or pressure pulses originating from short and intense laser excitation [3] our technique for pressure step generation [4] makes use of the transient response of a piezoelectric crystal plate. In the experimental arrangement, c.f. Fig. 1, a 100 nsec duration, 400 volt pulse from a 50ת-coaxial cable discharge generator is applied to the

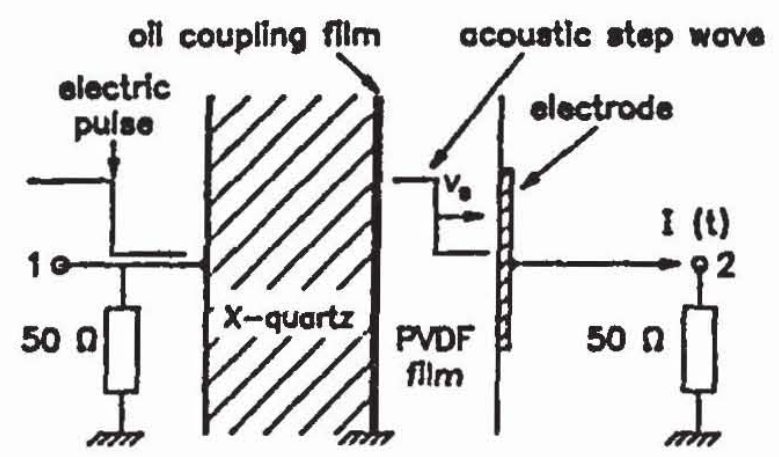

Fig. 1

Schematic representation of the experimental technique. (1) Connection to voltage pulse generator (150psec risetime), (2) Connection to wideband preamplifier and oscilloscope.

$\mathrm{X}$-quartz plate of $3 \mathrm{~mm}$ thickness and $25 \mathrm{~mm}$ diameter. The sharp voltage step (risetime < $1 \mathrm{nsec}$ ) at $t=0$ causes an initial state of homogeneous stress in the piezoelectric crystal, with zero stress boundary condition for the acoustically unloaded crystal. This initial condition gives rise to the propagation of zero stress step waves from both surfaces into the crystal. Corresponding to the electric field switch-off after 100 nsec rectangular 


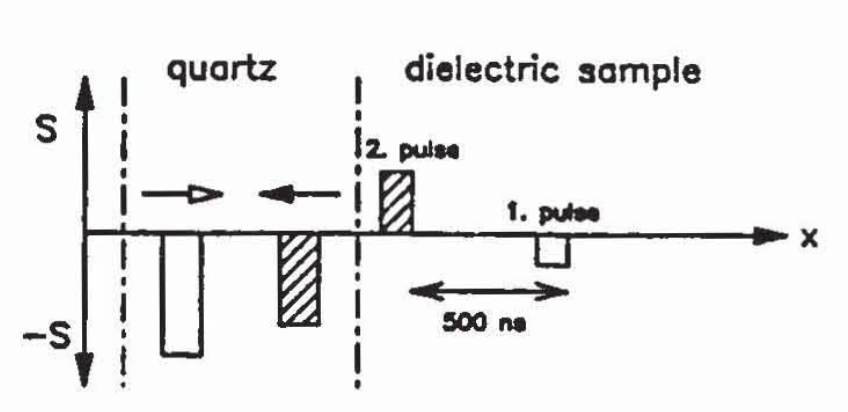

Fig. 2

Acoustic pulse sequence radiated into the sample by the $X$-quartz plate transient response. The second pulse leading-edge is used as step wave.

velocity (pressure) pulses are generated by the voltage pulse, Fig. 2. By mechanical contact of a dielectric film to one crystal surface a sequence of corresponding velocity (pressure) pulses with alternating sign is radiated into the sample. The first pulse is directly radiated into the dielectric film by the motion of the crystal surface in contact with the sample with no time delay. The second signal with inverted sign is caused by the pulse starting at the acoustically free crystal rear side. Since the amplitude of this signal transmitted into the sample amounts to twice the amplitude of the first pulse, all measurements are performed with the leading edge of this pulse as acoustic step wave. In addition, electrical disturbances from the cable discharge and the acoustical reflections in the sample have decayed within the acoustical delay time of 500nsec. The sequence of further multiple reflected and decaying pulses can be ignored in the measurement. Risetime limitations resulting from the finite quartz disc capacitance, the inertia loading of the $X$-quartz crystal and the sample by evaporated metal electrodes can be kept below 1 nsec with Al-layers not exceeding $1 \mu \mathrm{m}$ thickness on the quartz crystal and $0.1 \mu \mathrm{m}$ thickness on the dielectric film sample. Mostly the dielectric film sample has not been metallized since it appeared sufficient to use the quartz crystal grounding electrode also as the sample grounding electrode, whereas the sample detecting electrode consisted of a conducting rubber disc with 3 to $5 \mathrm{~mm}$ diameter. As coupling liquid between quartz and sample silicone oil has been used. Applying moderate static pressure to the rubber electrode the acoustic contact to the quartz plate is sufficiently good, i.e. the oil coupling film is squeezed to a thickness of 100 to $200 \mathrm{~nm}$, avoiding the risetime deteriorating influence of dust particles by the softness of the dielectric film and the conducting rubber electrode. The influence of acoustic attenuation leading to a risetime increase of the step wave during propagation, in samples of 100um thickness can be reduced by cooling to liquid nitrogen temperatures. 
Step wave response signal and evaluation

As measurements with a thin quartz disc as detector have verified, it is easy to calibrate the generator in terms of the calculated acoustic step wave velocity or pressure amplitude [4] radiated into the sample. Thus, the acoustic step wave velocity amplitude in the sample is known from the measured generator pulse voltage. The observed step wave short circuit current response $I_{S}(t)$ [4] can be expressed by charge, polarization and "true" piezoelectricity distributions in the dielectric sample:

$$
I_{s}(t)=A v / L\left[(1+\gamma)\left(\sigma_{1}+\int_{0}^{x_{s}-v_{s} t} \rho(x) d x\right)-e\left(x_{s}\right)-\gamma P_{0}\left(x_{s}\right)\right]
$$

with $I_{S}(t)$ signal current under short circuit conditions, c.f. Fig. $1 ; x_{S}=v_{S} t$ step front position after propagation time $t ; A=$ sample area; $L=$ sample thickness; $\mathrm{v}=2 \mathrm{Z}_{\mathrm{s}} \mathrm{e}_{\mathrm{xx}} \mathrm{v}_{\mathrm{p}}\left[\left(\mathrm{z}_{\mathrm{s}}+\mathrm{z}_{\mathrm{q}}\right) \mathrm{z}_{\mathrm{q}} \mathrm{L}_{\mathrm{q}}\right]^{-1}$ velocity amplitude in the step wave with $\mathrm{Z}_{S}, \mathrm{z}_{Q}=$ acoustic impedance of the sample and the quartz plate respectively, $e_{x x}=$ piezoelectric constant of quartz, $L_{Q}=$ thickness of the quartz plate, $v_{p}=$ applied pulse voltage; $v_{s}=$ step propagation velocity; $\gamma=-1 / \varepsilon \partial \varepsilon / \partial s$ relative electrostriction coefficient;

$$
\sigma_{1}=1 / \int_{0}^{L} P_{0}(x) d x-\int_{0}^{l}(1-x / L) \rho(x) d x
$$

static counter charge per area on the grounding electrode of the sample; $\rho(x)=$ density of true excess charges in the sample volume coupled to the particle velocity of the step wave; $e_{33}\left(x_{S}\right)=$ intrinsic piezoelectric constant, i.e. changes of elementary dipolemoment by strain; $P_{0}\left(x_{S}\right)$ persistent polarization in the unstrained film, note the elementary dipolemoments of this contribution do not change under strain. In deriving Eq. 1 Maxwells equations and the strain relation in the step wave $s=-v / v_{s}$ have been used. For the homogeneous strained sample, i.e. complete step transit, we obtain from Eq. 1 the total charge transport:

$$
Q=\int_{0}^{\omega_{0}} I_{s}(t) d t=A s / L \int_{0}^{L}\left[e(x)-P_{0}(x)\right] d x
$$

in agreement with the expectation that the charge distribution $\rho\left(x_{S}\right)$ and electrostriction do not contribute to the homogeneous piezoelectricity. In contrast, the transient response $I_{S}(t)$ with $e\left(x_{S}\right)=0$ and $P_{0}\left(x_{S}\right)=0$ allows for the determination of the charge distribution by

$$
\rho(x)=d I_{s} / d t L\left[A v v_{s}(1+\gamma)\right]^{-1}
$$

as follows from Eq. 1. Alternatively, a pure $P_{0}(x)$ distribution, i.e. $e\left(x_{S}\right)=0$ and $\rho\left(x_{S}\right)=0$ results in 


$$
I_{s}(t)=A v / L\left[(1+\gamma) \sigma_{1}-\gamma P_{0}\left(x_{s}\right)\right]
$$

This indicates that $\mathrm{P}_{\mathrm{O}}\left(\mathrm{x}_{\mathrm{S}}\right)$ can be resolved only by finite electrostriction $\gamma \neq 0$; the $\sigma_{1}$ contribution results in a positive constant current during the step transit, thus the $P_{O}\left(x_{s}\right)$ signal is negative with a constant positive background signal resulting from $\sigma_{1}$. With $\rho\left(x_{S}\right)=0$ and $e\left(x_{s}\right)=0, P_{0}\left(x_{s}\right)$ follows from the short circuit current response according to:

$$
P_{0}\left(x_{s}\right)=(A v \gamma)^{-1}\left[(1+\gamma) v_{s} \int_{0}^{t} I_{s}(t) d t-L I_{s}(t)\right]
$$

$t_{L}=$ acoustic transit time for the sample with thickness $L$ and $x_{s}=y_{s} t$. We finally discuss the situation of complete field neutralization by the condition $\rho(x)=\partial P / \partial x$, i.e. the charge distribution is in electrostatic equilibrium with the persistent polarization. In this case, Eq.1 with finite $\gamma$ reduces to

$$
I_{s}(t)=A v / L\left[P_{0}\left(x_{s}\right)-e\left(x_{s}\right)\right]
$$

where $P_{0}\left(x_{S}\right)$ can be replaced by

$$
\int_{0}^{x} \rho(x) d x
$$

which corresponds to the physical interpretation that the origin of $I_{S}(t)$ lies in the movement of charges and not in the movement of elementary dipoles with constant dipolemoment. Electrostriction, i.e. $\gamma \neq 0$ does not enter in this case since the internal macroscopic field is zero by field neutralization, i.e. finite conductance of the dielectric material. With respect to the relative electrostriction coefficient entering the evaluation for pure charge or polarization distributions, a simple experimental determination is possible by applying a static DCvoltage $V_{B}$ to the sample still under dynamic short circuit conditions. The resulting rectangular contríbution to the short circuit signal response

$$
I_{s}(t)=A v / L(1+\gamma) V_{B} / L \varepsilon \varepsilon_{0}
$$

allows for the evaluation of $\gamma$ with $\varepsilon$ known from dielectric measurements. Thus, the determination of $I_{S}(t)$ allows quantitative evaluation in terms of Eqs. 1, 5, 7, 8 if it is already known that either "pure" piezoelectricity, pure charge, pure polarization, or zero field conditions are valid.

Comparison with experiment

Especially with finite electrical conductance as in PVDF the condition of vanishing field by charge compensation 


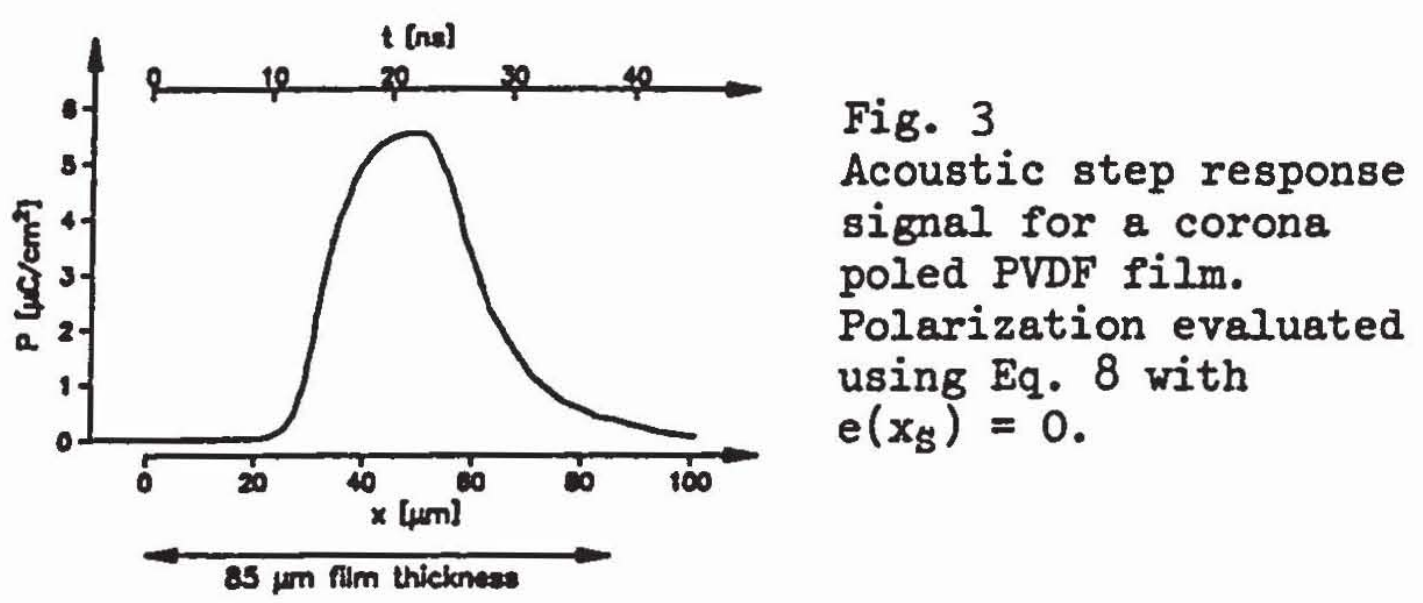

leads to the simple result of Eq. 8 by which $\rho\left(x_{s}\right)$ and $\mathrm{P}_{0}\left(x_{s}\right)$ can be calculated from the experimental $I_{s}(t)$ dependence if $e\left(x_{s}\right)$ is either known [5] or comparatively small, c.f. Fig. 3. For charge compensated internal polarization zones, as in Fig. 3, the absence of signal contributions at the film boundary is characteristic. In the experimental result, Fig. 3, the equilibrium or zero field condition in principle could also arise from local neutralization of the dipole field of polarized crystallites. This "counter charge" situation corresponds to a vanishing $\rho\left(x_{s}\right)$ and leads to a reduced signal amplitude [5]. Experimentally, for the sample of Fig. 3 the presence of space charges according to $\rho(x)=\partial P / \partial x$ has been verified by microtome slicing combined with charge measurement. Also the measured total charge flow during the poling process corresponds to the polarization measured by the acoustic step wave response. The build-up of counter charges in contrast would imply significantly higher total charge flow than observed. An experimental example for a pure charge distribution [6] is shown in Fig. 4. A PTFE sample of $25 \mu \mathrm{m}$ thickness has been

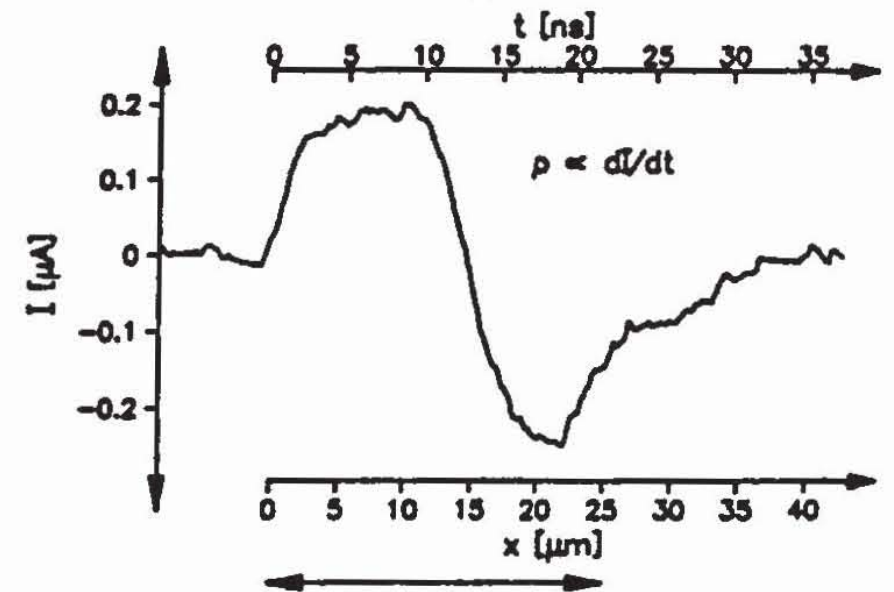

$25 \mathrm{~mm}$ fim thickness
Fig. 4 Acoustic step response signal for a $30 \mathrm{kV}$ electron beam charged [6] PTFE sample. Discussion see text. 
irradiated with electrons, producing a negative charge layer in the film. As expected the step wave signal begins with positive constant current caused by the positive counter charge on the grounding electrode and changes abruptly to the negative as the step wave passes the real charge layer. Quantitative evaluation according to Eq. 5 requires the knowledge of $\gamma$ which can be obtained by the additional measurement discussed before.

Time evolution of charge and polarization

Our step wave technique, so far, has been discussed with respect to the investigation of already prepared samples. In some situations it is of interest to study the buildup of charge and polarization distributions during the poling procedure. This can be accomplished by inserting an additional insulating film, e.g. mylar between the conducting rubber electrode and the sample film. The insulating film acts as a series capacitor reducing the signal amplitude with no influence on the signal shape. Poling of the sample is possible by DC-current (voltage) supply with a thin metal electrode at the insulating film evaporated on the face in sample contact. The same technique is used for measuring the electrostriction coefficient. In both cases quantitative evaluation additionally requires the measurement of the insulating film capacitance.

Financial support by the Deutsche Forschungsgemeinschaft is gratefully acknowledged.

\section{References}

1. For a general review see Electrets (Edited by G.M. Sessler) Topics in Applied Physics, Vol. 33. Springer Verlag, Berlin (1980).

2. P. Laurençeau et al., Phys. Rev. Lett. 38, 46 (1977)

3. G.M. Sessier, J.E. West, R. Gerhard Phys. Rev. Lett. 48, 563 (1982)

4. W. Eisenmenger, M. Haardt, Solid State Communications, 41, 917 (1982)

5. M.G. Broadhurst et al., J. Appl. Phys. 49,4992 (1978)

6. To be published in co-operation with G.M. Sessler and R. Gerhard having also prepared the PTFE sample. 